\title{
Reducing Ultrasonic Signal Noise by Algorithms based on Wavelet Thresholding
}

\author{
M. Kreidl, P. Houfek \\ Traditional techniques for reducing ultrasonic signal noise are based on the optimum frequency of an acoustic wave, ultrasonic probe \\ construction and low-noise electronic circuits. This paper describes signal processing methods for noise suppression using a wavelet \\ transform. Computer simulations of the proposed testing algorithms are presented.
}

Keywords: ultrasonic testing, wavelet transform, thresholding of wavelet coefficients, de-noising algorithms.

\section{Introduction}

Ultrasonic non-destructive testing is a versatile technique that can be used in a wide variety of materials analysis applications. There are several sources of noise that hide a fault. The difficulties of ultrasonic methods result from strong signal attenuation, caused mainly by scattering at the inhomogeneities in the structure of the material. The decreasing transmitted ultrasonic signal causes strong coherent noise. The optimum frequency of an acoustic wave provides the highest signal-to-noise ratio compatible with the detection of a specific discontinuity. Each combination of discontinuity type and material may have a different optimum frequency. Noise occurs on contact between the probe and the material, and finally noise is caused by the electronics that are used. This noise can totally mask even large backwall echoes. The basic characteristics of an ultrasonic instrument and probe are their sensitivity and resolution. Sensitivity is the characteristic of ultrasonic testing that determins the ability to detect small signals limited by the signal-to-noise ratio. Resolution is the ability of an ultrasonic flaw detection system to give separate indications of discontinuities that have almost the same range and/or lateral position.

\section{Theoretical}

Reduction of the $\mathrm{S} / \mathrm{N}$ ratio using algorithms of wavelet thresholding is described in this paper. The method is based on replacing small wavelet coefficients by zero, and keeping or shrinking the coefficients with an absolute value above the threshold discrete wavelet transform (hard or soft thresholding) [1]. The wavelet procedure consists of three steps: multiple-level decomposition on approximation and detail coefficients (DWT - Discrete Wavelet Transform), thresholding of detail coefficients, reconstruction (IDTW - Inverse Discrete Wavelet Transform).

The choice of the threshold is relevant for noise reduction. The thresholding methods proposed in this research were the following:

- rigsure - quadratic loss function for a soft threshold estimate of the risk for a particular threshold value,
- sqtwolog - the threshold is set to a fixed value, which is computed as the square root of the logarithm of the discrete values of the signa,l

- heursure - a mixture of the previous options,

- minimaxi-the threshold value is calculated for the minimum of the mean square error against an ideal procedure.

In the Matlab environment (Wavelet Toolbox) the following types of waves were used for the signal decomposition to the approximation coefficient and detail coefficient: Daubechies, Symlet, Coiflet, Biorthogonal pairs.

The model of the classical high frequency signal as an A-scan with a probe frequency of $20 \mathrm{MHz}$ and an equivalent sampling frequency of $256 \mathrm{MHz}$ was implemented from real recorded data by computer for testing of algorithms. Noise at different levels was added to this signal and the noise-signal ratio was expressed by the coefficient of the noise level NSR (1), which is, according to Equation (1):

$$
\mathrm{NSR}=20 \log \left(\frac{N_{\text {ef }}}{S_{\text {ef }}}\right)
$$

where $N_{\text {ef }}$ is RMS (root mean square) value of noise,

$$
\mathrm{S}_{\mathrm{ef}} \quad \mathrm{RMS} \text { value of signal. }
$$

The NSR rate was chosen for better understanding of the following graphs.

The coefficient of noise reduction $\delta \mathrm{SN}$ was defined for a comparison of the success of the used algorithms, according to Eq. (2):

$$
\delta \mathrm{SN}=20 \log \left(\frac{\frac{S_{\mathrm{ef} 1}}{N_{\mathrm{ef} 1}}}{\frac{S_{\mathrm{ef} 2}}{N_{\mathrm{ef} 2}}}\right) \quad[\mathrm{dB}]
$$

where $N_{\text {ef1 }}$ is RMS value of noise included in signal,

$N_{\text {ef2 }} \quad$ RMS value of noise included in signal, after application of a given algorithm,

$S_{\text {ef1 }} \quad$ RMS value of signal corresponding to the fault echo,

$S_{\text {ef2 }} \quad$ RMS value of signal after application of a given algorithm. 

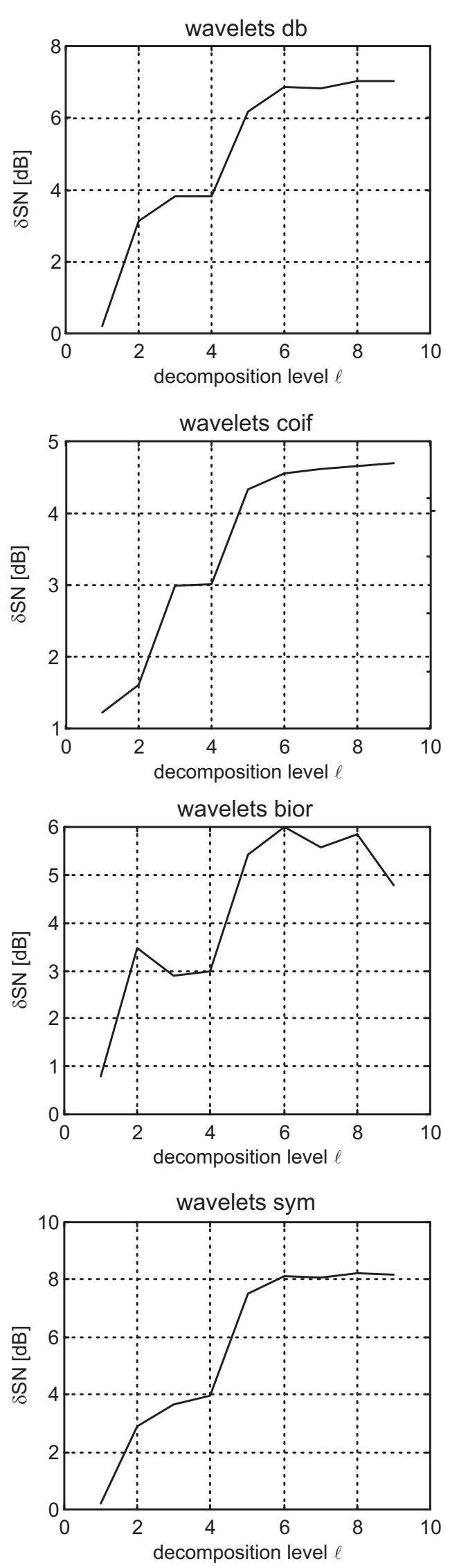

Fig. 1: The average dependence of noise reduction on decomposition level $\ell$ for particular kinds of wavelets
The RMS values of the simulated signal were obtained from digital samples corresponding to fault echoes before and after application of a given algorithm.

While analysing the signal it is convenient to set the extraction of approximations and details of the given signal to a certain level $\ell$. Fig. 1 shows a graph of noise reduction in simulated signal as a function of the decomposition level $\ell$ (e.g., the maximum order of used approximations and details). The noise-signal ratio expressed by coefficient NSR (1) was chosen as $-20 \mathrm{~dB}$.

This picture shows that the influence of the decomposition level is roughly indicated at a value of $\ell=6$ in all cases, and higher values are not proper. A value of $\ell=6$ was chosen for the maximum decomposition level (i.e., the maximum level of the used approximations and details) according the analysis of the ultrasonic signal.

The four above thresholding methods were applied to the simulated signal with coefficient NSR $=-20 \mathrm{~dB}$ after DWT analysis (with $\ell=6$ ), with a steady threshold at particular levels of analysis. The wavelets in the table were chosen empirically. The resulting effect of the noise, expressed as a value of the coefficient $\delta \mathrm{SN}$ for different kinds of the waves and thresholding methods, is shown in Tab. 1.

Results for waves that gave the change in the noise-signal ratio expressed by $\delta \mathrm{SN}$ smaller than $10 \mathrm{~dB}$ are not presented in this table. These waves probably have a different wave shape from the echoes contained in ultrasonic signal, and therefore they are not suitable for noise reduction.

Tab. 1: Values of noise reduction coefficient $\delta S N[d B]$ for different types of waves and thresholding

\begin{tabular}{|l|c|c|c|c|}
\hline & \multicolumn{4}{|c|}{ Thresholding method } \\
\hline Type of wave & rigrsure & heursure & sqtwolog & minimaxi \\
\hline db4 & 21.59 & 23.73 & 27.07 & 26.91 \\
\hline db6 & 19.37 & 20.59 & 26.42 & 27.42 \\
\hline db7 & 18.52 & 22.64 & 25.05 & 23.39 \\
\hline db8 & 20.90 & 25.61 & 28.24 & 27.43 \\
\hline db9 & 18.14 & 19.55 & 26.93 & 25.70 \\
\hline db10 & 18.84 & 21.53 & 26.63 & 26.32 \\
\hline bior 1.3 & 11.55 & 11.48 & 28.28 & 28.76 \\
\hline bior3.9 & 18.98 & 22.55 & 29.81 & 27.26 \\
\hline bior 4.4 & 18.63 & 22.95 & 27.59 & 26.48 \\
\hline coif1 & 17.61 & 17.47 & 25.29 & 21.55 \\
\hline coif2 & 17.88 & 17.94 & 25.55 & 22.14 \\
\hline coif4 & 18.99 & 23.62 & 26.10 & 23.35 \\
\hline sym3 & 17.59 & 22.15 & 27.51 & 25.81 \\
\hline sym4 & 20.82 & 20.84 & 26.89 & 25.22 \\
\hline sym5 & 19.54 & 19.86 & 25.96 & 25.76 \\
\hline sym6 & 17.16 & 20.71 & 26.70 & 24.86 \\
\hline sym7 & 17.45 & 20.74 & 27.65 & 24.25 \\
\hline sym8 & 17.22 & 20.45 & 24.79 & 22.82 \\
\hline
\end{tabular}



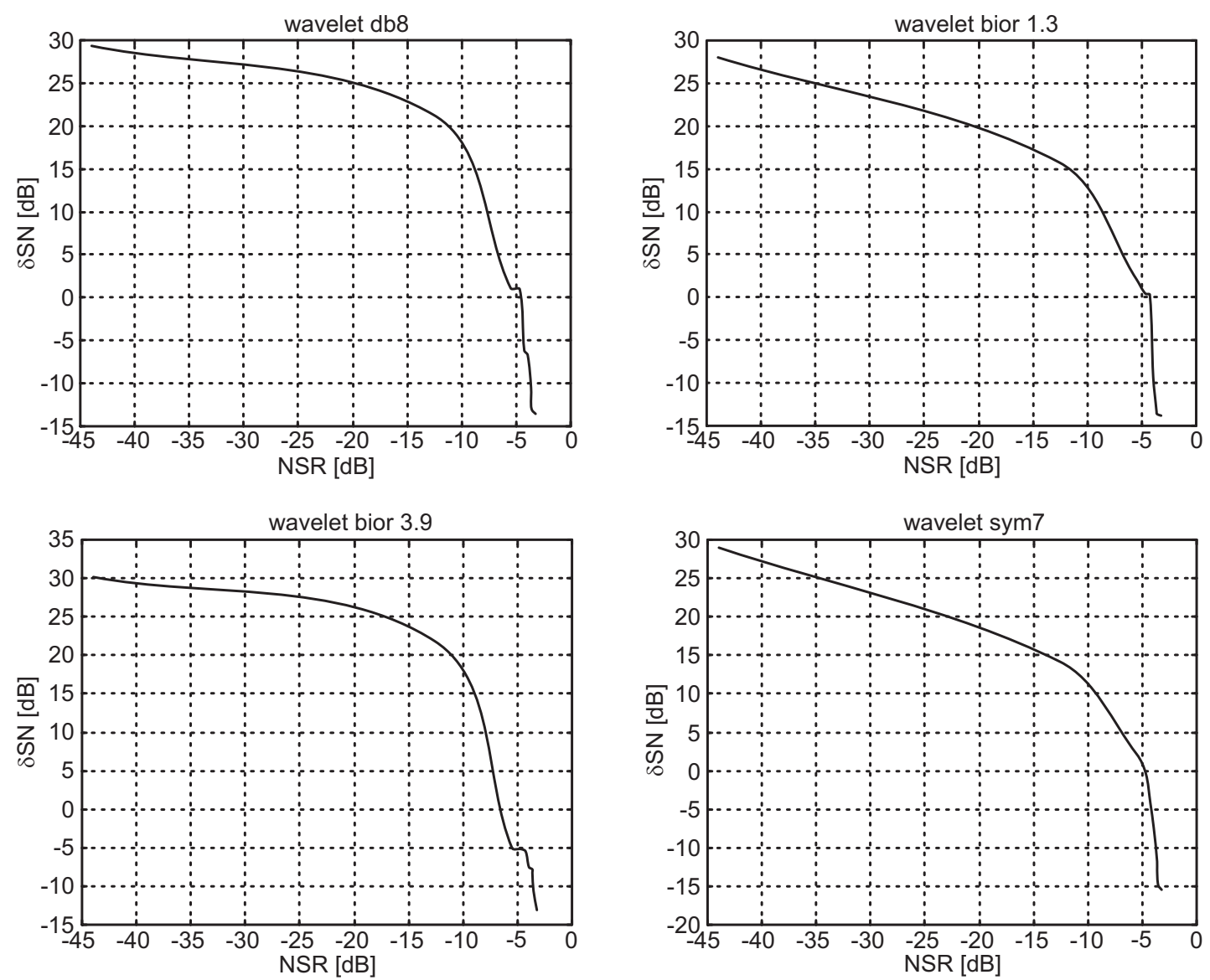

Fig. 2: Dependence of the noise reduction coefficient on the noise level in the signal for the four waves, with maximum noise reduction effect

The results presented in Table 1 show that the values of the noise reduction coefficient vary from 17 to $30 \mathrm{~dB}$. The different effects of different thresholding methods are also apparent. The "rigrsure" and "heursure" methods gave worse results than the other methods. The best results were obtained using the "sqtwolog" method. Only this method will therefore be considered from now on. The four waves "db8", "bior3.9", "bior 1.3", "sym3" which produced the highest values of coefficient $\delta \mathrm{SN}$ were chosen.

In following section the ultrasonic signal occurred with additive noise, which was generated on the basis of the model of real noise for the noise reduction rates simulated by computer. The noise-signal ratio was consequently increased in the ranges of the NSR coefficient from $-45 \mathrm{~dB}$ to $-3 \mathrm{~dB}$. A graph expressing the dependence of the noise reduction coefficient $\delta \mathrm{SN}$ on the coefficient of the noise level in the signal for the waves given above is shown in Fig. 2.

The dependence in Fig. 2 shows that the maximum value of the NSR coefficient, for which the $\delta S N$ value is positive, varies in the range from $-7,5 \mathrm{~dB}$ to $-4 \mathrm{~dB}$.

Until now we have considered only the standard thresholding methods for noise reduction.

Now a new method [2] will be presented. This involves computing the optimum threshold value individually for wavelet decomposition of every detail coefficients. For verification purposes, an analysis was made of the simulated signal, primarily up to the level $\ell=6$. This analysis was made using the "bior3.9" wave, i.e., the wave that gave best results in the prior analysis. Fig. 3 shows the details and approximations obtained during wavelet analysis of the signal with noise given an NSR coefficient value of $-7,15 \mathrm{~dB}$. The time curve of the analysed signal is displayed in Fig. 3.

Fig. 3 shows that the simulated echo is best displayed in detail No. 3 and particularly in detail No. 4, while other details are representations of the noise. These results show that it is necessary to perform maximum suppression of the noise part of the signal contained in all details, except details No. 3 and No. 4.

A simple algorithm was written in the Matlab environment to determine the optimum thresholds. This algorithm goes gradually through the combinations of threshold values for every detail of the signal in the range from 0 to 1 . After threshold processing for every combination of threshold reconstruction, the signal is made and the computation of the noise reduction coefficient $\delta \mathrm{SN}$ is processed.

In the following table, the optimum values are written for wave "bior 3.9", together with the value $\delta \mathrm{SN}$, which characterises the noise reduction. 
The time curve of the signal
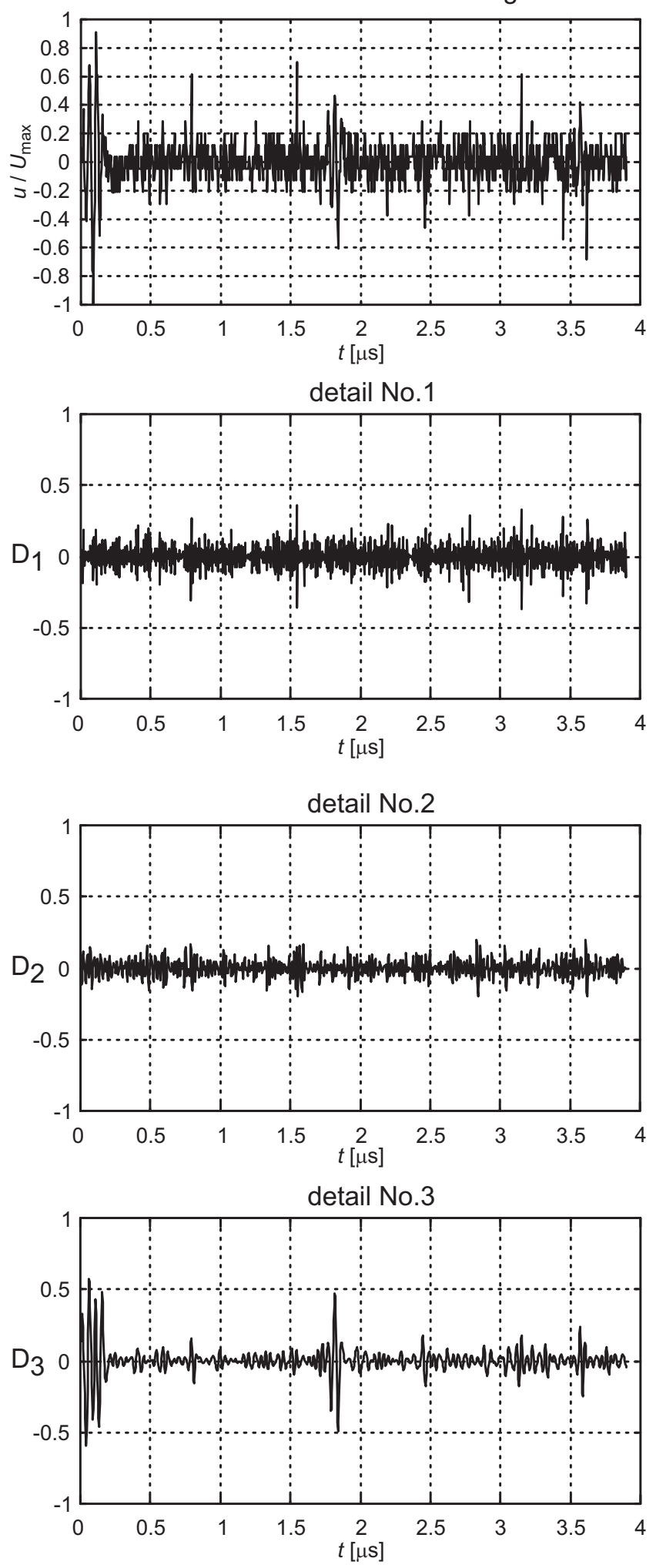

aproximation No.2

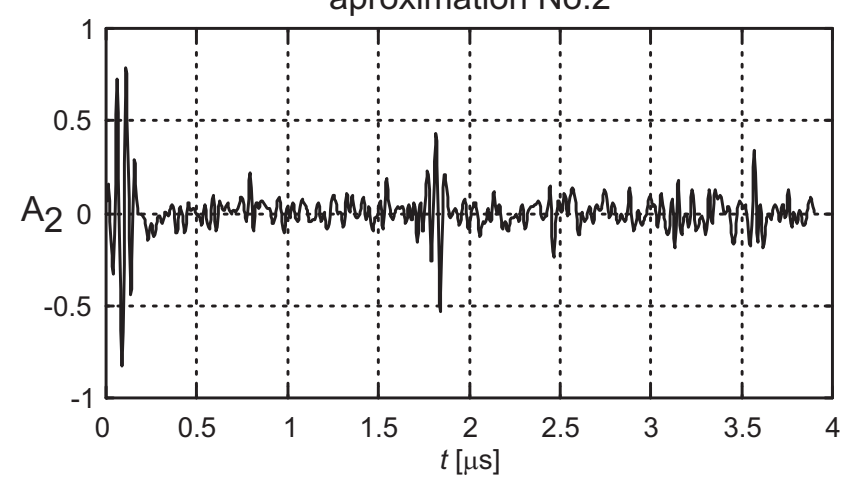

detail No.4
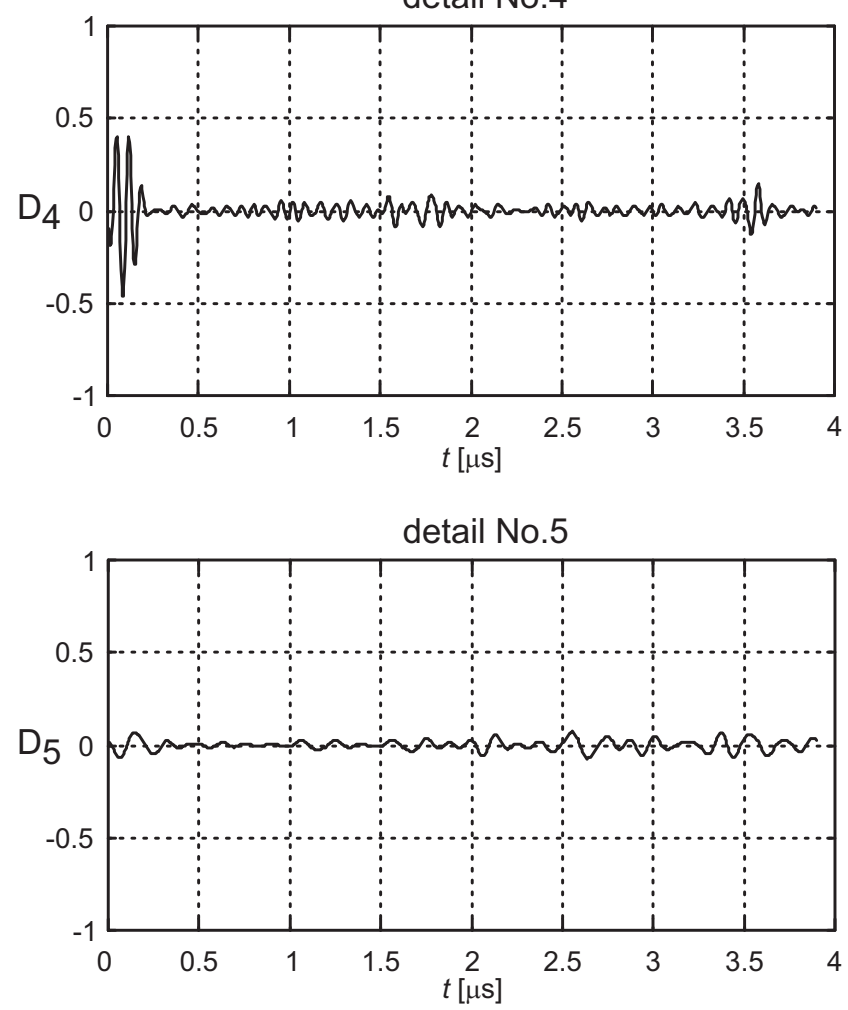

detail No.6

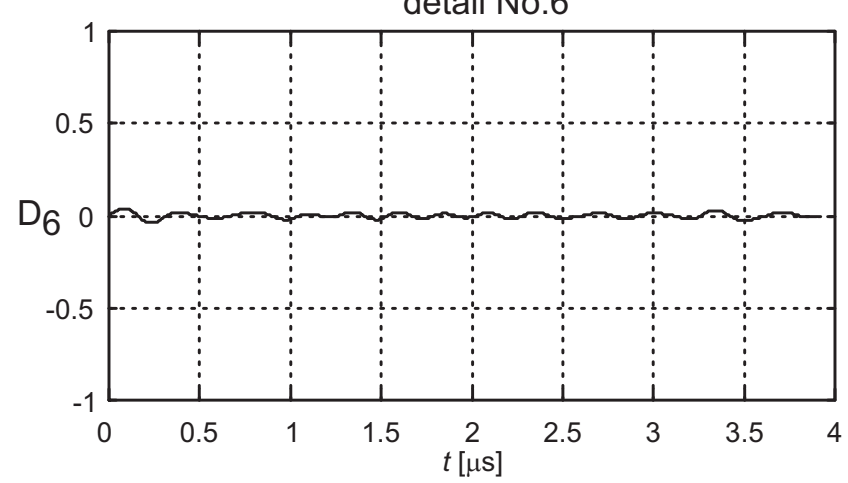

Fig. 3: Decomposition of the simulated signal for $\ell=6$ (drawings of all details of the wavelet analysis and one selected approximation)

Tab. 2: Optimum choice of thresholds for maximum noise reduction

\begin{tabular}{|c|c|c|c|c|c|c|}
\hline \multicolumn{6}{|c|}{ Levels of signal details } & \multirow[t]{3}{*}{$\delta \mathrm{SN}[\mathrm{dB}]$} \\
\hline detail & detail & detail & detail & detail & detail & \\
\hline No. 1 & No. 2 & No. 3 & No. 4 & No. 5 & No. 6 & \\
\hline 0.5 & 0.3 & 0.2 & 0.1 & 0.1 & 0.3 & 26.5 \\
\hline
\end{tabular}

An analysis was then made of the influence of changes in the particular thresholding levels. After this analysis had been performed in the Matlab environment, a program was written which gradually shifted the threshold values of the detail and computed the noise reduction effect. The levels of the other details were set to the optimum value according to Tab. 2 . The 

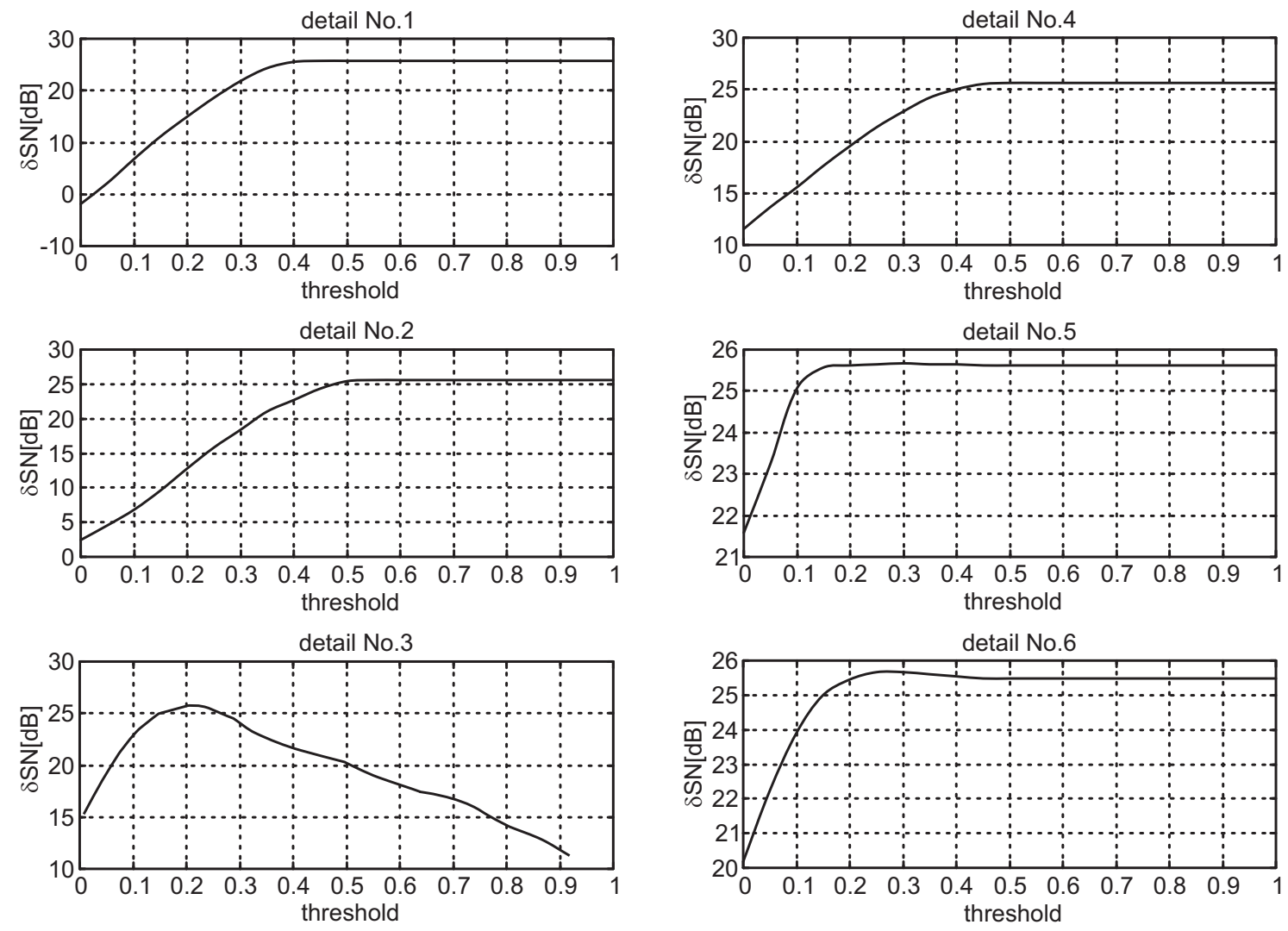

Fig. 4: Influence of threshold values on signal noise reduction

results of this analysis for wave "bior3.9" are drawn in Fig. 4. The dependence of the noise reduction coefficient on the threshold value increases before settling at a particular value, for all details except detail No. 3. Since details No. 1, No. 2, No. 4, No. 5 and No. 6 include mostly noise (see Fig. 3), it may be assumed that a maximum reduction will be achieved if we totally cut out the discrete values of these details. Fig. 3 also shows that the settling details of the higher levels are faster. This is because the discrete values of the particular details are decreasing and total removal is achieved immediately with lower threshold levels.

If we focus on the dependence of $\delta \mathrm{SN}$ on the level of threshoding of detail No. 3, we will see that this dependence has a certain maximum representing the optimum noise reduction. The existence of this maximum can be explained as follows:

Detail No. 3 contains information concerning the fault echo, but it also contains a certain noise value. If we cut down the noise value, then the noise from the signal will be reduced. The amplitude of the fault echo will also be reduced, but the main influence is from the noise reduction effect. If we increase the threshold value so that the noise is totally removed, then after the next reduction the fault echo will be reduced and the value of coefficient $\delta \mathrm{SN}$ will decrease.

A comparison of the value of coefficient $\delta \mathrm{SN}$ received after applying the new proposed thresholding method with the value achieved using standard thresholding methods provides the following result: the value of coefficient $\delta \mathrm{SN}$

is about $20 \mathrm{~dB}$ higher when the new thresholding method is used.

\section{Experimental}

The proposed algorithm based on applying wavelet transformation was then tested on real data [2]. The measurement was done on a special gauge, made of two metal sheets 9,2 $\mathrm{mm}$ in thickness, which were jump welded. A fault was created artificially on one part of the scale by a $0,5 \mathrm{~mm}$ hole, which was manufactured by spark technology. The results of the measurements are given in Fig. 5.

\section{Conclusion}

Our analysis indicates that all waves from the sym family are suitable for noise reduction in an ultrasonic signal, followed by coiflet and symlet - type waves and some chosen biorthogonal pairs (especially "bior3.9" or "bior 4.4"). Noise reduction for these waves is characterised by noise reduction coefficient $\delta \mathrm{SN} \geq 12 \mathrm{~dB}$. On the other hand, it is not recommended to use "db1", "db5", "bior3.1" to "bior3.7" and "coif5" waves. These waves have a different shape [4] from the echoes in an ultrasonic signal.

We then investigated the different thesholding methods with respect to noise reduction. The standard methods indicate that no optimum algorithm exists. Our proposed method produced the best results, consisting of the optimum 

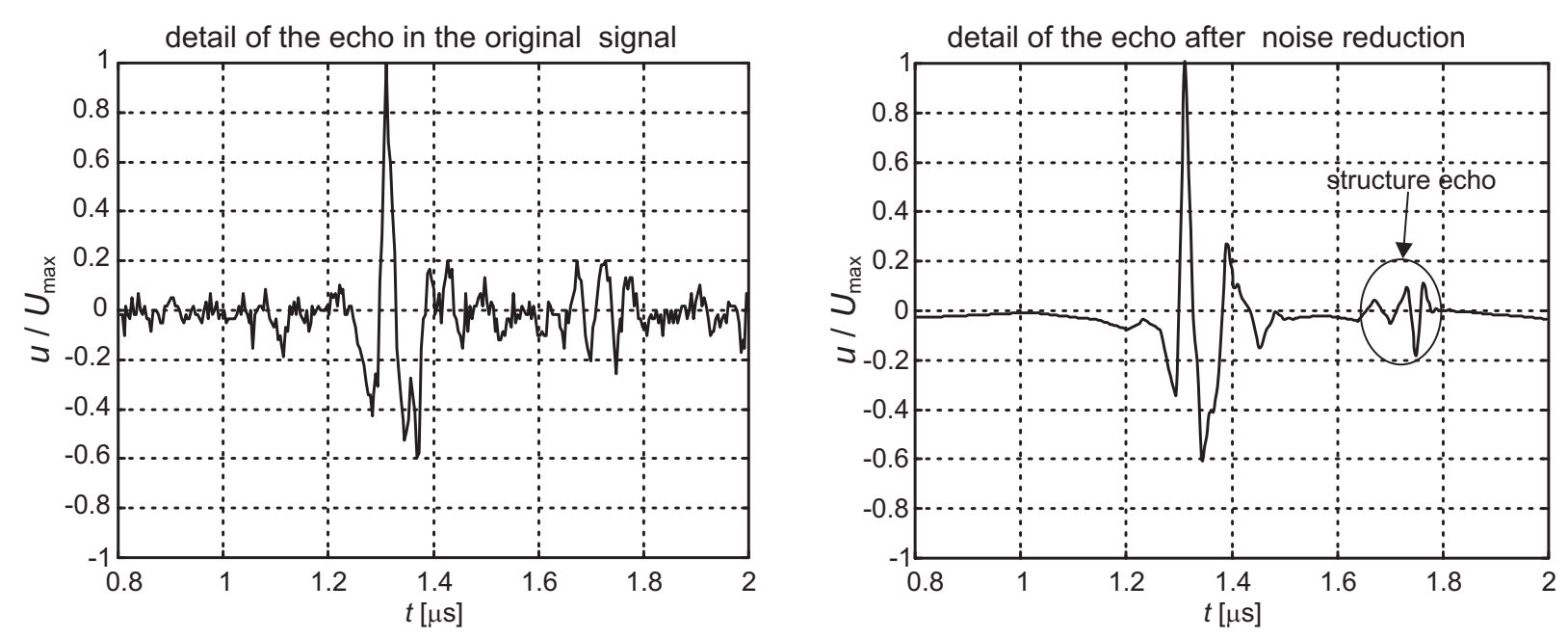

Fig. 5: Results of noise reduction, using the proposed new algorithm with wave "sym3"

threshold value individually for a wavelet analysis of each detail coefficient of the signal. By choosing a suitable threshold, the noise reduction coefficient can be increased by about $9 \mathrm{~dB}$ on the real signal, and by even $10 \mathrm{~dB}$ on a simulated signal.

\section{Acknowledgement}

This research work has received support from research program No J04/98:210000015 „Research of New Methods for Physical Quantities Measurement and Their Application in Instrumentation" of the Czech Technical University in Prague (sponsored by the Ministry of Education, Youth and Sports of the Czech Republic).

\section{References}

[1] Donoho, D. L.: De-noising by Soft-Thresholding. IEEE Transactions on Information Theory, 1995, Vol. 41, No. 3, p. 613-627.

[2] Houfek, P.: Metody zvyšováni citlivosti a rozlišovaci schopnosti v ultrazvukové defektoskopii. [Methods for improving sensitivity and recognition in ultrasonic defectoscopy].
Dizertační práce, Fakulta elektrotechnická ČVUT, Praha, 2001.

[3] Kreidl, M. et al: Diagnostické systémy. [Diagnostic Systems]. Monografie, Praha: Vydavatelství ČVUT, 2001, p. 123-128.

[4] Misiti, M., Misiti, Y., Oppenheim, G., Poggi, J. M.: Wavelet Toolbox User's Guide. Natick (USA): The Math Works, 1966.

[5] Strang, G., Nguyen, T.: Wavelets and Filter banks. Wellesley (USA): Wellesley-Cambridge Press, 1966.

Ing. Petr Houfek, Ph.D.

e-mail: houfek@fel.cvut.cz

Doc. Ing. Marcel Kreidl, CSc.

e-mail: kreidl@fel.cvut.cz

Department of Measurement

Czech Technical University in Prague

Faculty of Electrical Engineering

Technická 2

16627 Prague 6, Czech Republic 